\title{
An Enhanced Cooperative Spectrum Sensing with Wavelet Denoising and Softened Hard Decision for Cognitive Radio Networks
}

\author{
G. Padmavathi ${ }^{1}$ and S. Shanmugavel ${ }^{2}$ \\ ${ }^{1}$ Department of Electronics and Communication Engineering, Sri Venkateswara \\ College of Engineering, Sriperumbudur, Tamilnadu, India \\ ${ }^{2}$ Department of Electronics and Communication Engineering, Anna University, \\ Chennai, Tamilnadu, India \\ gpadma@svce.ac.in.sedhusvel@gmail.com
}

\begin{abstract}
Cognitive Radio (CR) is a novel technology that can improve the utilization efficiency of the radio spectrum and also address the spectrum scarcity problem by opportunistically using the unused channels (spectrum holes / white spaces) in the Licensed Spectrum of Primary Users. Spectrum sensing is the primary function of CR which determines the spectrum holes for opportunistic usage by Secondary Users. Spectrum sensing plays a critical role in Cognitive Radio. The development of a reliable and accurate spectrum sensing is very essential to propound quick and accurate detection of white spaces for better throughput of Secondary User network under the constraint of the specified Primary System protection. In this paper, a Cooperative Spectrum Sensing using Wavelet Denoising along with Soften Hard Decision technique has been proposed to improve the performance of spectrum sensing. Simulation result shows an improvement of nearly $15 \%$ in the Probability of Missed Detection for a target Probability of False Alarm of 0.1 and SNR of $-10 \mathrm{~dB}$. Also 50\% improvement in error probability has been achieved with 1-D one level wavelet denoising.
\end{abstract}

Keywords: Cognitive Radio, Cooperative Spectrum Sensing, Wavelet Denoising, Softened Hard Decision

\section{Introduction}

Electromagnetic spectrum is a limited natural resource, access to which is regulated by government agencies like Federal Communications Commission (FCC) in the United States. In the traditional approach to spectrum management, a major portion of it has been statically allocated to many of the existing wireless applications for their exclusive operations. Wireless communications have experienced booming growth in the recent years and because of the fixed spectrum assignment policy there is no spectrum available for the new upcoming wireless applications.

On the other hand, recent survey by Spectrum Policy Task Force (SPTF) within FCC [1] indicated that many licensed frequency bands are not used for significant periods of time with an average duty cycle of only $13 \%$ indicating under utilization of the spectrum. Cognitive Radio (CR) is a promising technology to address the problems of spectrum scarcity and under utilization [2]. Spectrum sensing, the most critical and foundation function of CR, identifies the unused licensed frequency bands that are not used by the licensed users known as Primary Users (PUs) for opportunistic access by Secondary Users (SUs). 
Sensing performance is crucial to the performance of both Primary and Secondary Networks which is determined using the metrics Probability of Detection $\left(\mathrm{P}_{\mathrm{d}}\right)$ and Probability of False Alarm $\left(\mathrm{P}_{\mathrm{f}}\right)$. $\mathrm{P}_{\mathrm{d}}$ denotes the probability of a CR User declaring that a PU is present when the spectrum is indeed occupied by the PU and $P_{f}$ denotes the probability of a CR user declaring that a PU is present when the spectrum is actually free.

Many factors such as multipath fading, shadowing, and the receiver uncertainty problem [3] significantly degrades the detection performance in spectrum sensing. Cooperative Spectrum Sensing (CSS) [4-6] is an attractive and effective approach to combat multipath fading and shadowing and mitigate the receiver uncertainty problem. The sensing performance is enhanced in CSS by exploiting the spatial diversity in the observations of spatially located CRs. CRs share their sensing information for making a combined decision more accurate than the individual decisions [4].

The classification of CSS, based on how the cooperating CRs share the sensing data in the network, is centralized, distributed and relay-assisted as shown in Figure 1 [7].

Centralized CSS has been used in this work in which a central entity called Fusion Center (FC) coordinates all the activities related to spectrum sensing - selecting the channel for sensing, instructing the cooperating CRs to perform local sensing, combing the results of local sensing from individual CRs to make a final decision and reporting the decision back to the cooperating CRs.
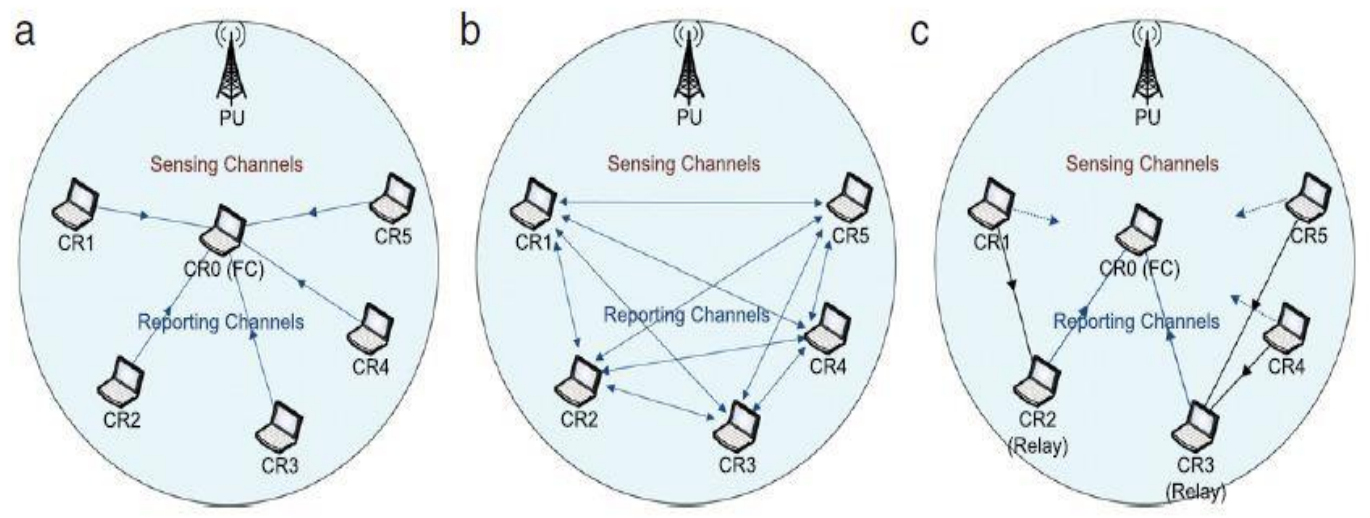

Figure 1. Classification of Cooperative Sensing: (a) Centralized, (b) Distributed and (c) Relay-assisted

\section{Energy Detection Based Spectrum Sensing with Double Threshold and Softened Hard Decision}

The spectrum sensing for primary signal detection can be formulated as a binary hypothesis-testing problem:

$$
\begin{array}{ll}
y(t)=n(t), & H 0: \text { primary user is absent; } \\
y(t)=h(t) \cdot x(t)+n(t), & H 1: \text { primary user is in operation }
\end{array}
$$

Where $y(t)$ represents received signal at CR,

$\mathrm{x}(\mathrm{t})$ represents transmitted signal by PU,

$h(t)$ is the channel gain of the sensing channel between the PU and the CR,

$\mathrm{n}(\mathrm{t})$ is the zero - mean additive white Gaussian noise(AWGN) 
The energy detection method is optimal for detecting any unknown zero-mean constellation signal [4]. In energy detection approach, the radio frequency energy in the channels or the received signal strength indicator (RSSI) is measured in a fixed bandwidth ' $\mathrm{W}$ ' over an observation time window ' $\mathrm{T}$ ' to determine whether the channel is occupied or not. The received energy is compared with the threshold value to make a decision about the presence or absence of the PU.

The output of energy detector is given by

$$
\mathrm{E}=\sum_{i=1}^{M}\|Y(i)\|^{2}
$$

Assuming that the noise uncertainty in the wireless environment is described as $\left[1 / \rho \sigma_{\mathrm{n}}{ }^{2}\right.$, $\rho \sigma_{\mathrm{n}}^{2}$ ], where $\rho>1$ is a parameter that quantifies the size of the uncertainty. In the proposed double threshold decision, the lower threshold $\lambda_{1}$ is selected according to the minimum noise variance, and the upper threshold $\lambda_{2}$ is selected according to the maximum noise variance [8]. Therefore

$$
\begin{aligned}
& \lambda_{1}=2 / \rho \sigma_{\mathrm{n}}^{2} \Gamma^{-1}\left(\mathrm{u}, \mathrm{P}_{\mathrm{f}} \Gamma(\mathrm{u})\right) \\
& \lambda_{2}=2 \rho \sigma_{\mathrm{n}}{ }^{2} \Gamma^{-1}\left(\mathrm{u}, \mathrm{P}_{\mathrm{f}} \Gamma(\mathrm{u})\right)
\end{aligned}
$$

where $\Gamma(\cdot)$ is the gamma function, $\Gamma^{-1}(\cdot, \cdot)$ denotes the inverse of the incomplete gamma function, $\mathrm{u}$ is time bandwidth product.

The local sensing decision rule is defined with two thresholds as follows:

$$
\mathrm{LD}= \begin{cases}0, & \mathrm{E} \leq \lambda_{1} \\ , & \lambda_{2}<\mathrm{E}<\lambda_{1} \\ 1, & \mathrm{E} \geq \lambda_{2}\end{cases}
$$

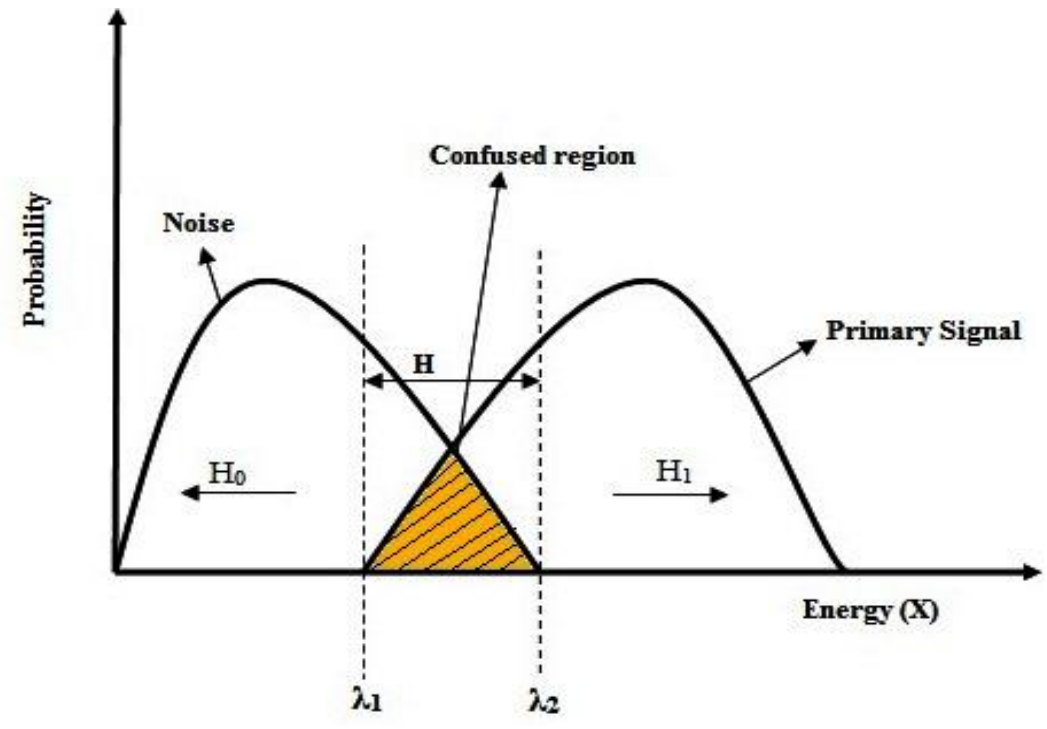

Figure 2. Energy Distribution Graph of PU Signal and Noise Demonstrating the Confused Region 


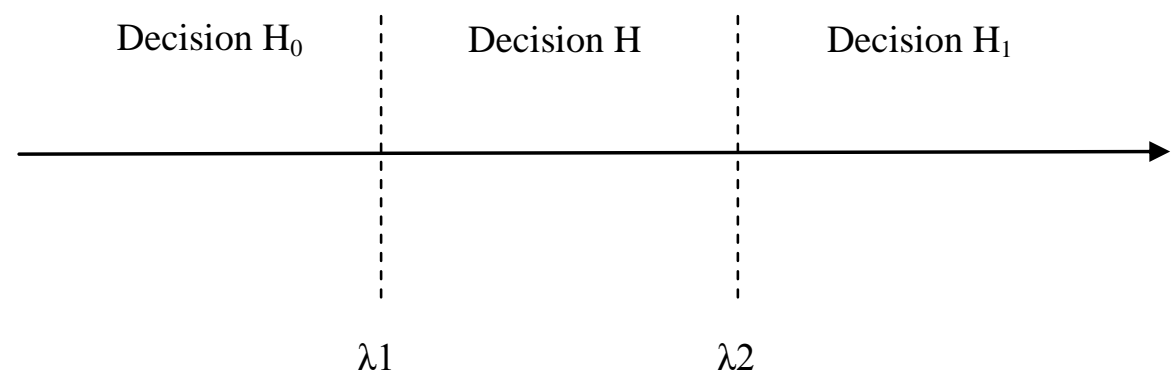

Figure 3. Double Threshold Decision Logic

The region between $\lambda 1 \& \lambda 2$, known as the confused region, is divided into four equal subregions as shown below by calculating $\mathrm{p}[8]$.

$\mathrm{p}=($ upper threshold $\lambda 1-$ lower threshold $\lambda 2) / 4$.

The four quantizing intervals are $\lambda 1 \mathrm{~A}, \mathrm{AB}, \mathrm{BC} \& \mathrm{C} \lambda 2$ such that

$$
\mathrm{A}=\lambda 1+\mathrm{p}, \mathrm{B}=\mathrm{A}+\mathrm{p} \& \mathrm{C}=\mathrm{B}+\mathrm{p} \text {. }
$$

The corresponding two bit quantized values for the sub regions are indicated in Figure 4.

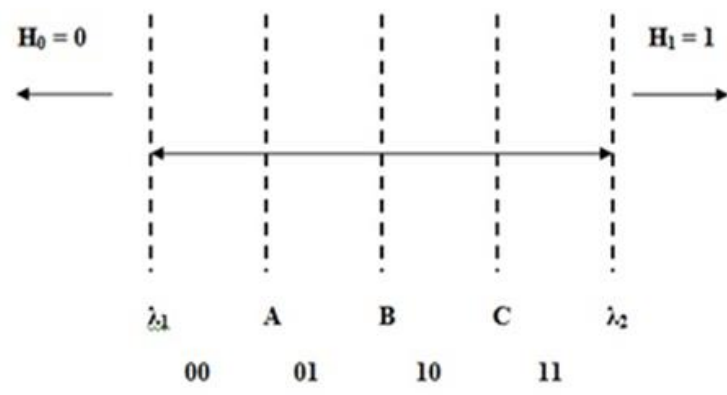

Figure 4. Sub-regions of Confused Region

The sensing performance is measured in terms of probability of detection $\mathrm{P}_{d}$ or the probability of missed detection $P_{m}$ which is $\left(1-P_{d}\right)$ and probability of false alarm $P_{f}$.

Probability of detection $\left(\mathrm{P}_{\mathrm{d}}\right)$ is defined under hypothesis $\mathrm{H} 1$ as the probability of correctly detecting the presence of the primary signal.

Probability of false alarm $\left(\mathrm{P}_{\mathrm{f}}\right)$ is defined under hypothesis $\mathrm{H} 0$ as the probability of falsely declaring the presence of primary signal.

$$
\begin{aligned}
& \mathrm{P}_{\mathrm{d}}=\mathrm{P}\{\text { decision }=\mathrm{H} 1 \mid \mathrm{H} 1\}=\mathrm{P}\{\mathrm{Y}>\lambda \mid \mathrm{H} 1\} \\
& \mathrm{P}_{\mathrm{f}}=\mathrm{P}\{\text { decision }=\mathrm{H} 1 \mid \mathrm{H} 0\}=\mathrm{P}\{\mathrm{Y}>\lambda \mid \mathrm{H} 0\}
\end{aligned}
$$

From the primary user perspective, higher the probability of detection better will be the primary user protection. From the secondary user perspective, lower the probability of false alarm better will be the opportunity for unlicensed access.

\section{Wavelet Denoising}

Wavelet transform is widely used in denoising signal processing applications. The Continuous Wavelet Transform (CWT) is provided by Eq. (5), where $y(t)$ is the signal to be analyzed, $\psi(t)$ is the mother wavelet or the basis function[9]. 


$$
Y_{\text {WT }}(a, b)=\frac{1}{\sqrt{|a|}} \int y(t) \bullet \Psi^{*}\left(\frac{t-b}{a}\right) d t
$$

The Discrete Wavelet Transform (DWT) is a sampled version of the CWT. The DWT is computed by low pass and high pass filterings of the discrete time-domain signal as shown in Figure 1. The signal is denoted as $X[n]$, where $\mathrm{n}$ is an integer. The low pass filter is denoted by $G_{0}$ while the high pass filter is denoted by $H_{0}$. At each level, the high pass filter produces detail information $d[n]$, while the low pass filter associated with scaling function produces coarse approximations $a[n]$.

\subsection{Spectrum Sensing with 1-D Wavelet Denoising}

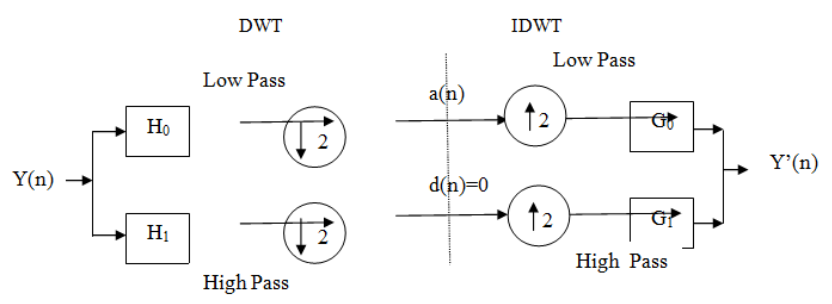

Figure 5. 1-D One Level Wavelet Transformation

A CR node has to detect $\mathrm{M}$ consecutive sampling points in the band of a licensed user each time.

$$
\begin{array}{lll}
\mathrm{Y}[\mathrm{i}]= & \mathrm{N}[\mathrm{i}], & \mathrm{H} 0 \\
& \mathrm{~h} * \mathrm{X}[\mathrm{i}]+\mathrm{N}[\mathrm{i}], & \mathrm{H} 1
\end{array}
$$

where $\mathrm{Y}$ [i], $\mathrm{X}$ [i] and $\mathrm{N}[\mathrm{i}$ ] are the signal received at $\mathrm{CR}$, signal transmitted by PU and the noise of the $i$-th sampling point respectively and ' $h$ ' is the channel gain.

The objective of energy sensing is to decide whether $\mathrm{H} 0$ or $\mathrm{H} 1$ is true by sensing the energy of the signal $\mathrm{Y}$ which is given by (2).

As energy sensing requires very short detection period, the channel gain and primary user's signal are supposed to have few changes during each detection period. So, the system model in Eq. (6) can be simplified [10] as:

$$
Y[i]= \begin{cases}N[i] & H_{0} \\ x+N[i] & H_{1}\end{cases}
$$

Wavelet denoising is useful mainly based on the "concentrating" ability of wavelet transform. The signal always has its energy concentrated in a small number of wavelet dimensions and the noise spreads its energy over a large number of coefficients. Eq. (7) can be written in wavelet transform domain as [9]

$$
\left[a_{y}, d_{y}\right]=W Y=W(X+N)=W X+W N
$$

Where $W$ denote a left invertible wavelet transformation matrix of the discrete wavelet transform (DWT), $X$ equals $[x, x, \ldots, x]$ or a $\mathrm{M}$ length zero vector. Since $\mathrm{X}$ has few changes, 
the detail information of WX is nearly zero. So the detail information $d_{y}$ only contains the detail information of noise in the wavelet transformation domain. After removing this detail information, the desired signal can be retrieved by the inverse wavelet transform without any loss of the original signal X; while the noise energy is significantly lowered. Higher SNR yields better sensing performance. A good SNR gain has been achieved with 1-D WD as indicated in Table1.

Table 1. SNR Gain with 1-D One Level Wavelet Denoising

\begin{tabular}{|l|l|l|}
\hline Input SNR in dB & Output SNR in dB & SNR Gain \\
\hline-16.1206 & 0.1489 & 16.2705 \\
\hline-10.8933 & 0.4869 & 11.3849 \\
\hline-5.1971 & 1.6731 & 6.8694 \\
\hline 1.8068 & 5.6811 & 3.8770 \\
\hline
\end{tabular}

The procedure of spectrum sensing with 1-D wavelet denoising [10] is summarized as follows:

i. Calculate the discrete wavelet transform coefficients of signal $Y=[y 1, y 2, \ldots, y M]$ and get the detail information $d_{y}$ and coarse approximation $a_{y}$.

ii. Set the detail information vector $d_{y}=0$, calculate the inverse wavelet transform with coarse approximation $a_{y}$ and new detail information $d_{y}$, and get the new signal $Y^{\prime}$.

iii. Calculate the energy of the new signal $Y .^{\prime}$

iv. Compare energy with the threshold values $\lambda_{1} \& \lambda_{2}$ and make a decision to send $0\left(\mathrm{H}_{0}\right.$ - Absence of PU) or $1\left(\mathrm{H}_{1}\right.$ - Presence of PU ) or two bit quantized values $(\mathrm{H})$.

\section{System Architecture}

The architecture of the system for the proposed CSS is shown in the following Figure 6.

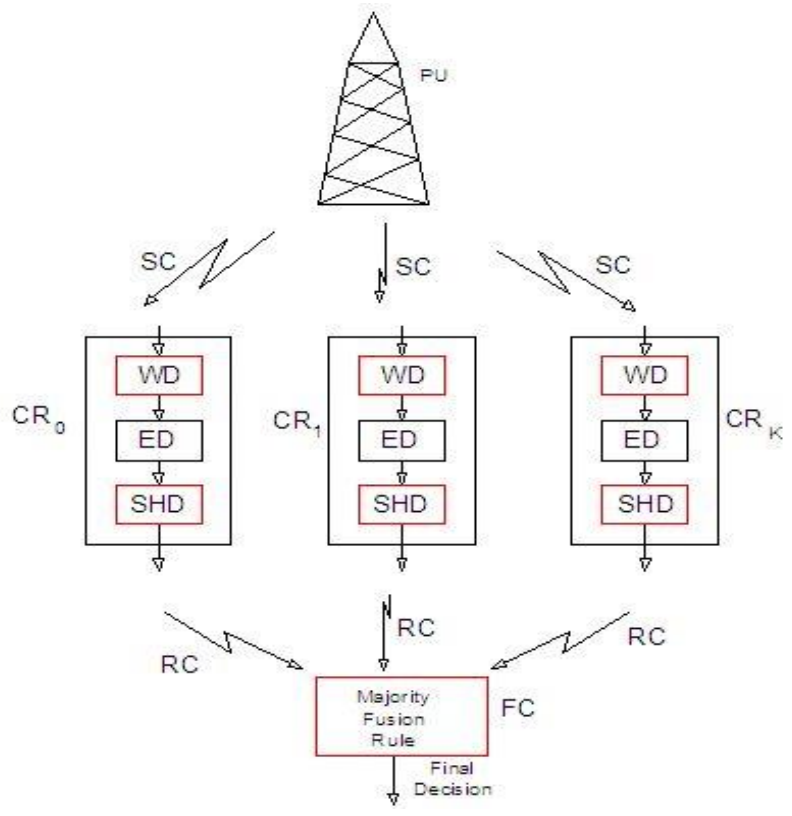

Figure 6. CSS Architecture with WD and SHD 


\subsection{Detection Procedure at Individual CRs and Fusion Center}

If the energy of the received denoised signal is less than $\lambda_{1}$, a bit 0 is sent to indicate the absence of PU. If the energy is greater than $\lambda_{2}$, a bit 1 is sent to indicate presence of PU. Otherwise two bit quantized value, depending on the region in which the energy falls, is sent. The FC Counts for the received No. of 0s (C0), 1s (C1), 00s (C00), 01s (C01), 10s (C10) $\& 11 \mathrm{~s}$ (C11). It also computes the total No. of local decisions in the confused region as $\mathrm{C}=$ $\mathrm{C} 00+\mathrm{C} 01+\mathrm{C} 10+\mathrm{C} 11$. Without loss of generality the the No. of decisions in region0 is added to the No. of decisions on $\mathrm{H}_{0}$ (absence hypothesis) and in region 3 to $\mathrm{H}_{1}$ as $\mathrm{C} 0=\mathrm{C} 0+\mathrm{COO}$ and $\mathrm{C} 1=\mathrm{C} 1+\mathrm{C} 11$. If $\mathrm{C} 01=\mathrm{C}$, then $\mathrm{C} 1=\mathrm{C} 1+\mathrm{C} 01$ and If $\mathrm{C} 10 \geq \mathrm{C} / 2$, then $\mathrm{C} 1=\mathrm{C} 1+\mathrm{C} 10$. Finally, If $\mathrm{C} 1>\mathrm{C} 0$, then decision is "PU is present"; Otherwise decision is "PU is absent"

\subsection{Simulation Results}

Simulations were carried out using MAT Lab Version 7.10.0(R2010 a) with the parameters shown in Table 2.

Table 2. Simulation Parameters

\begin{tabular}{|l|l|}
\hline PU Signal & Gaussian Distributed Signal \\
\hline Noise & AWGN \\
\hline SNR & $-10 \mathrm{~dB}$ \\
\hline No. of simulation runs & 1000 \\
\hline $\begin{array}{l}\text { Probability of received energy falling } \\
\text { between upper \& lower thresholds under } \\
\mathrm{H}_{1},\end{array}$ & 0.01 \\
\hline Size of noise uncertainty, $\rho$ & 1.01 \\
\hline No. of samples & 1000 \\
\hline
\end{tabular}

Figure 8 shows the Receiver Operating Characteristic (ROC) Curves for both theoretical \& simulated cases. The result shows an improvement of $15 \%$ in $P_{d}$ for a $P_{f}$ of 0.1 . Figure 9 indicates the Complementary Receiver Operating Characteristic (CROC) Curves for both theoretical \& simulated cases. The Probability of error $\left(\mathrm{P}_{e}=\mathrm{P}_{\mathrm{m}}+\mathrm{P}_{\mathrm{f}}\right)$ has been plotted in Figure 10 for various values of SNR ranging from -30 to $5 \mathrm{~dB}$. Nearly $50 \%$ reduction in error probability is observed for an SNR of $-30 \mathrm{~dB}$.

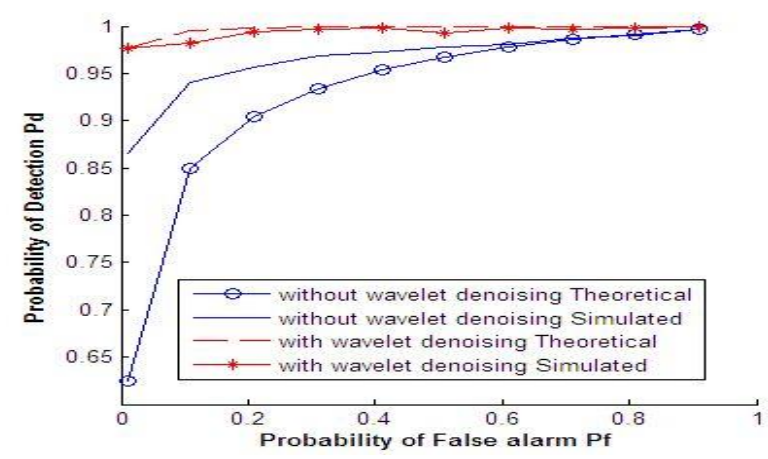

Figure 7. ROC Plot $\left(\mathbf{P}_{\mathrm{f}}\right.$ vs $\left.\mathbf{P}_{\mathrm{d}}\right)$ 


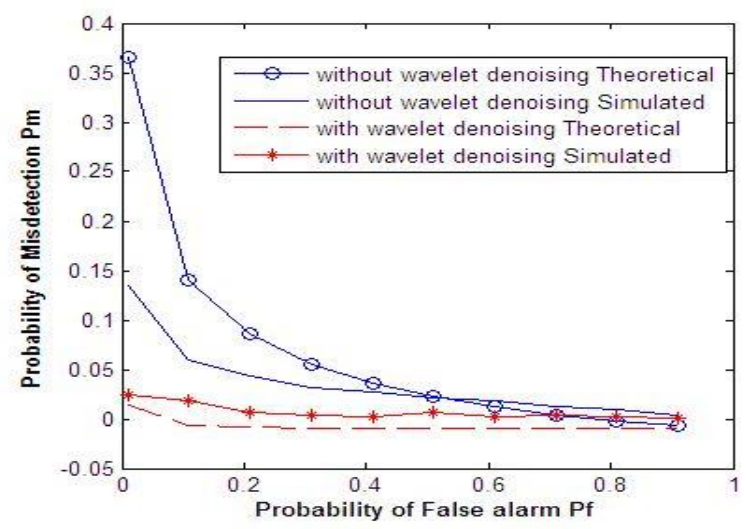

Figure 8. CROC Plot $\left(P_{f}\right.$ vs $\left.P_{m}\right)$

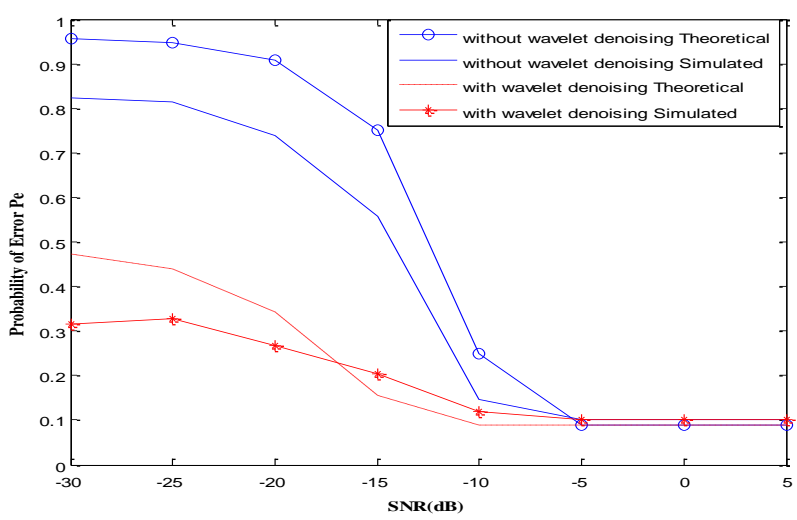

Figure 9. Error Probability Plot (SNR vs Pe)

\section{Conclusion}

The performance of the proposed cooperative spectrum sensing with wavelet denoising and softened hard decision technique has been analyzed through ROC curves. The plots exhibit an improvement of nearly $15 \%$ in $\mathrm{P}_{\mathrm{d}}$ for a target $\mathrm{P}_{\mathrm{f}}$ of 0.1 in case of theoretical. A very good SNR gain, especially at low SNRs (16.2705 at -16 dB), has been achieved with 1-D one level Wavelet denoising. The error probability plot shows a reduction of $50 \%$ with wavelet denoising at SNR of $-30 \mathrm{~dB}$.

\section{References}

[1] Federal Communications Commission, "Spectrum Policy Task Force”, Rep. ET Docket no. 02-135, (2002).

[2] M. S. Haykin, "Cognitive radio: brain-empowered wireless communica-tions", IEEE Trans. Communication, vol. 23, no. 2, (2005).

[3] I. F. Akyildiz, W.-Y. Lee, M. C. Vuran, and S. Mohanty, "NeXt generation/dynamic spectrum access/cognitive radio wireless networks: A survey", ELSEVIER J. Computer Networks, vol. 50, (2006).

[4] D. Cabric, S. M. Mishra and R. W. Brodersen, "Implementation issues in spectrum sensing for cognitive radios", in Proc. Asilomar Conference on Signals, Systems, and Computers, (2004).

[5] A. Ghasemi and E. S. Sousa, "Collaborative spectrum sensing for opportunistic access in fading environments", in Proc. First IEEE Symposium on Dynamic Spectrum Access Networks (DySpan'05), (2005) Baltimore, USA. 
[6] K. B. Letaief and W. Zhang, "Cooperative Communications for Cognitive Radio Networks", Proceedings of the IEEE, vol. 97, no. 5, (2009).

[7] I. F. Akyildiz, B. F. Lo and R. Balakrishnan, "Cooperative spectrum sensing in cognitive radio networks: A survey" in ELSEVIER, Physical Communication, vol. 4, (2011).

[8] S.-Q. Liu, B.-J. Hu and X.-Y. Wang, "Hierarchical Cooperative Spectrum Sensing Based on Double Thresholds Energy Detection", IEEE Communications letters, vol. 16, no. 7, (2012).

[9] Rioul and M. Vetterli, "Wavelets and sigal processing”, IEEE Signal Processing Mag., (1991) October.

[10] H. Wang, Y. Xu and X. Su, "Cooperative Spectrum Sensing with Wavelet Denoising in Cognitive Radio", IEEE $71^{\text {st }}$ Vehicular technology conference, (2010) May.

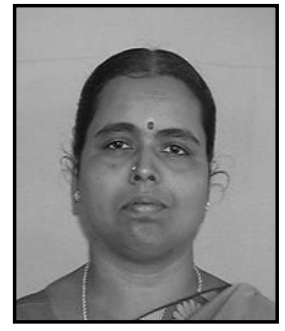

\section{Authors}

G. Padmavathi, she graduated from Thiagarajar College of Engineering, Madurai Kamaraj University, India in Electronics and Communication Engnieering in 1984. She obtained post graduation from Brila Institue of Technology and Science, Pilani, India in the specialization of Electronics and Control in 1993 and from Anna University in the specialisation of Communication Networks in 2006. She is working as Professor in ECE Dept., Sri Venkateswara College of Engineering, Sriperumbudur, India since 1993. Her current research focus is on spectrum sensing, spectrum management and cross layer optimization for cognitive radio networks. She is also interested in $4 \mathrm{G}$ wireless networks. She has published many technical papers in reputed journals and conferences.

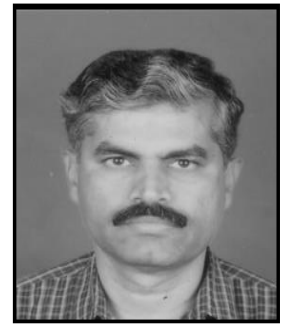

S. Shanmugavel, he graduated from Madras Institute of Technology, Chennai, India in Electronics and Communication Engineering in 1978. He obtained his Ph.D. degree in the area of coded communication and spread spectrum techniques from Indian Institute of Technology (IIT), Kharagpur, India in 1989. He joined as faculty of the Department of Electronics and Communication Engineering at IIT, Kharagpur, as a Lecturer in 1987 and became an Assistant Professor in 1991. Presently, he is a Professor in the Department of Electronics and Communication Engineering, College of Engineering, Anna University, Chennai, India. He has published more than 68 research papers in national and international conferences and 25 research papers in journals. He has been awarded the IETE-CDIL Award in September 2000 for his research paper. His areas of interest include Grid computing, heterogeneous networks, mobile ad hoc networks, ATM networks, Cognitive Radio Networks and CDMA engineering. 
International Journal of Future Generation Communication and Networking Vol. 7, No. 6 (2014) 